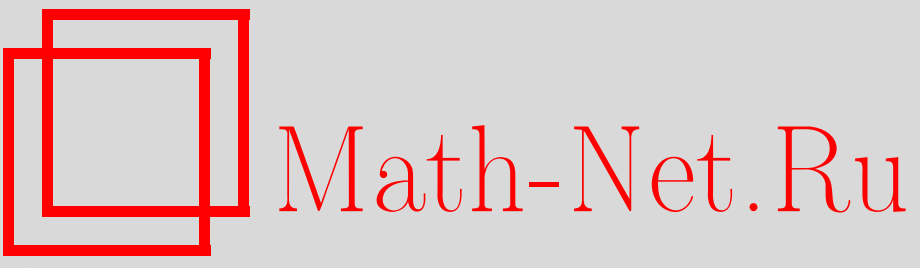

A. L. Kazakov, Construction and investigation of exact solutions with free boundary to a nonlinear heat equation with source, Mat. Tr., 2019, Volume 22, Number 2, 5475

DOI: https://doi.org/10.33048/mattrudy.2019.22.204

Use of the all-Russian mathematical portal Math-Net.Ru implies that you have read and agreed to these terms of use

http : //www . mathnet.ru/eng/agreement

Download details:

IP : 34.227 .88 .159

April 26, 2023, 07:04:53 


\title{
ПОСТРОЕНИЕ И ИССЛЕДОВАНИЕ ТОЧНЫХ РЕШЕНИЙ СО СВОБОДНОЙ ГРАНИЦЕЙ НЕЛИНЕЙНОГО УРАВНЕНИЯ ТЕПЛОПРОВОДНОСТИ С ИСТОЧНИКОМ
}

\author{
А. Л. Казаков
}

Статья посвящена построению и исследованию точных решений со свободной границей нелинейного параболического уравнения второго порядка. Решения относятся к классам обобщенно-автомодельных и обобщенных бегущих волн, их построение сводится к задачам Коши для обыкновенных дифференциальных уравнений (ОДУ) второго порядка, для которых доказаны теоремы существования и единственности решений. Проведен качественный анализ ОДУ путем перехода к динамической системе, построения и исследования ее фазового портрета, выполнены геометрические иллюстрации.

Ключевые слова и фразы: нелинейное уравнение теплопроводности с источником, тепловая волна, точное решение, теорема существования, качественный анализ обыкновенных дифференциальных уравнений.

\section{Введение}

Работа посвящена исследованию нелинейного (квазилинейного) уравнения с частными производными параболического типа

$$
T_{t}=\operatorname{div}(K(T) \nabla T)+L(T) .
$$

Здесь $T(t, \mathbf{x})$ - температура среды в момент времени $t$ в точке $\mathbf{x} \in \mathbb{R}^{3}$; $\operatorname{div}$ и $\nabla$ - операторы дивергенции и градиента по пространственным переменным; $K(T) \geq 0$ - монотонная достаточно гладкая функция, определяющая коэффициент теплопроводности; $L(T)$ - непрерывная функция, определяющая источник (сток).

Поскольку функция $K(T)$ монотонная, в (1) можно сделать замену переменных $T=K^{-1}(U)=\phi(U)$ и привести уравнение к виду

$$
U_{t}=U \Delta U+F(U)(\nabla U)^{2}+\Phi(U),
$$

где

$$
F(U)=U \phi^{\prime \prime}(U) / \phi^{\prime}(U)+1, \quad \Phi(U)=L(\phi(U)) / \phi^{\prime}(U)
$$

(C) А. Л. Казаков; 2019 
Рассмотрим наиболее часто встречающийся в приложениях случай, когда $K(T)=\lambda T^{\sigma}$, где $\sigma>0, \lambda>0-$ константы; $L(T)=\mu T^{\theta}$, где $\theta \geq 1$ и $\mu-$ константы, $\mu<0$ при наличии стока, $\mu>0-$ источника. Тогда (2) примет вид

$$
U_{t}=U \Delta U+\frac{(\nabla U)^{2}}{\sigma}+\alpha U^{\beta}
$$

где $\alpha=\sigma \mu \lambda^{(1-\theta) / \sigma}, \alpha>0-$ источник, $\alpha<0-$ сток; $\beta=(\sigma+\theta-1) / \sigma$, если $\sigma>0, \theta \geq 1$, то $\beta \geq 1$. Уравнение (3) в отечественной литературе называют «уравнением нелинейной теплопроводности со степенной нелинейностью и источником (стоком)» [17], в зарубежной - "the generalized porous medium equation" [25]. Оно интересно как с математической точки зрения, будучи типичным представителем нелинейных уравнений математической физики, так и с точки зрения приложений, поскольку описывает различные процессы в задачах тепло- и массопереноса, теории горения и взрыва, фильтрации, химической кинетике, популяционной биологии (см., например, [15; 25]).

Интересной разновидностью решений (1) со свободной границей является тепловая волна, под которой понимается конфигурация, состоящая из двух гиперповерхностей: $T(t, \mathbf{x}) \geq 0$ (возмущенное решение) и $T(t, \mathbf{x}) \equiv 0$ (фоновое нулевое решение), непрерывно состыкованных вдоль некоторой достаточно гладкой гиперповерхности $\Gamma(t, \mathbf{x})=0$, определяющей фронт тепловой волны (тепловой фронт). Первые примеры решений типа тепловой волны, распространяющейся в абсолютно холодной среде (по нулевому фону) с конечной скоростью, впервые были приведены в статье Я. Б. Зельдовича и А. С. Компанейца [5]. Близкие результаты для задач фильтрации были вскоре получены Г. И. Баренблаттом [1]. Позднее, в исследованиях А. Ф. Сидорова и его учеников [19], рассматривались вопросы разрешимости в классе аналитических функций специальных краевых задач для уравнения теплопроводности, имеющих решения типа тепловой волны. Был предложен и развит оригинальный подход к исследованию задач с заданными краевым режимом и тепловым фронтом (метод специальных рядов). С использованием этой методологии был доказан ряд теорем, обеспечивающих существование и единственность локально-аналитических решений упомянутых краевых задач как в одномерной [22; 23], так и в неодномерной $[6 ; 8]$ постановках, в том числе с подвижной границей [7; 9]. В отдельных случаях аналитическое исследование было дополнено численным с использованием гранично-элементного [8; 22; 23]. Отметим, что в упомянутых в этом абзаце работах рассматривалось уравнение без источника (стока).

Для исследования свойств тепловых волн эффективным инструментом являются точные решения, так как они позволяют выйти за пределы ма- 
лой окрестности, в которой обычно удается доказать сходимость рядов. Так, в теории режимов с обострением, построенной в школе А. А. Самарского, точные (в частности, типа бегущей волны и автомодельные) решения применяются в качестве иллюстрирующих примеров [17]. В статье [11] получены новые классы точных решений нелинейного уравнения теплопроводности без источников (стоков), имеющих тип тепловой волны.

Данная работа является прямым продолжением исследований [11], проведенных нами ранее. Рассматривается более общая постановка задачи, предусматривающая наличие источника (стока) со степенной зависимостью последнего от температуры. Решения описываются с помощью функций, которые определяются из начальных задач для обыкновенных дифференциальных уравнений второго порядка с особенностью перед старшей производной. В отличие от ранее изученных случаев, уравнения, вообще говоря, не являются автономными. Тем не менее для рассмотренных задач удалось доказать теоремы существования и единственности нетривиальных решений. Проведено подробное исследование свойств тепловых волн, имеющих вид обобщенной бегущей волны, для чего выполнен переход к динамической системе и построены ее фазовые портреты при различных значениях входных параметров.

\section{§1. Постановка задачи}

Уравнение (3) при наличии пространственных симметрий может быть преобразовано к виду

$$
u_{t}=u\left(u_{\rho \rho}+\frac{\nu u_{\rho}}{\rho}\right)+\frac{u_{\rho}^{2}}{\sigma}+\alpha u^{\beta} .
$$

Здесь параметр $\nu$ принимает значения 0 в случае плоской, $1-$ круговой и 2 - сферической симметрии; $\rho=\left(\sum_{i=1}^{\nu+1} x_{i}^{2}\right)^{1 / 2}-$ расстояние до начала координат; $u(t, \rho)$ - искомая функция. Для уравнения (4) будем искать нетривиальные точные решения со свободной границей, имеющие тип тепловой волны, распространяющейся с конечной скоростью по холодному фону, т. е. удовлетворяющие условию

$$
\left.u\right|_{\rho=a(t)}=0,
$$

где $a(t)>0$ - достаточно гладкая функция. Линию $\rho=a(t)$ называют фронтом тепловой волнъ. На ней тепловая волна стыкуется с тривиальным решением $u \equiv 0$, при этом искомая функция, очевидно, непрерывна, а производные терпят разрыв.

Отметим, что на фронте тепловой волны в уравнении (4) обращается в нуль множитель перед старшей производной $u_{\rho \rho}$, что приводит к вырождению уравнения с понижением порядка. Такие математические объекты 
относят к неклассическим уравнениям математической физики [3]. K этому классу принадлежат также уравнения, не разрешенные относительно старшей производной [20], которые с учетом их специфики называют в литературе уравнениями соболевского типа $[4 ; 12 ; 18]$ или не типа Коши Ковалевской [21].

\section{§2. Построение точных решений}

Будем искать решение задачи (4), (5) в следующем виде:

$$
u=\psi(t) v(z), \quad z=\varphi(t, \rho),
$$

где $\psi(t)$ и $v(z)$ определяется при решении обыкновенных дифференциальных уравнений (ОДУ). Подобный подход к поиску решений иногда называют методом Клаксона - Крускалла [16; 24]. По сути дела он представляет собой обобщение классического метода разделения переменных.

Будем рассматривать следующие способы задания функции $\varphi(t, \rho)$ :

$$
\varphi_{1}(t, \rho)=\rho-a(t), \quad \varphi_{2}(t, \rho)=\rho / a(t) .
$$

Выбор обоснован тем, что это две наиболее естественные разновидности переменной $z$, при которых условие (5) переписывается как $v\left(z_{0}\right)=0$ : в первом случае $z_{0}=0$, во втором случае $z_{0}=1$.

Решения вида (6), (7) близки к классическим типам точных решений нелинейных уравнений математической физики: в первом случае прослеживается аналогия с бегущими волнами, во втором - с автомодельными решениями. Будем, следуя [16], называть решения (6) первого типа $\left(z=\varphi_{1}(t, \rho)\right)$ обобщенными бегущими волнами, второго $\left(z=\varphi_{2}(t, \rho)\right)-$ обобщенно-автомодельными.

Теперь сформулируем и докажем две основные теоремы данного раздела. Рассмотрим последовательно случаи обобщенно-автомодельного решения и обобщенной бегущей волны.

Теорема 1. Задача (4), (5) имеет обобщенно-автомодельные решения в следующих случаях:

1) $\nu=0,1,2, \beta=1, \varphi_{2}(t, \rho)=\rho /\left[C_{1} \exp \left(C_{2} t\right)\right], \psi(t)=C_{1}^{2} C_{2} \exp \left(2 C_{2} t\right)$, a $v(z)$ удовлетворяет задаче

$$
v v^{\prime \prime}+\frac{1}{\sigma}\left(v^{\prime}\right)^{2}+\left(z+\frac{\nu v}{z}\right) v^{\prime}+\left(\frac{\alpha}{C_{2}}-2\right) v=0, \quad v(1)=0
$$

2) $\nu=0,1,2, \beta \neq 1,2, \varphi_{2}(t, \rho)=\rho /\left(C_{3} t+C_{4}\right)^{\omega}$,

$$
\psi(t)=\omega C_{3}\left(C_{3} t+C_{4}\right)^{2 /(2-2 \beta)}, \quad \text { где } \omega=(\beta-2) /(2 \beta-2),
$$


a $v(z)$ удовлетворяет задаче

$$
v v^{\prime \prime}+\frac{1}{\sigma}\left(v^{\prime}\right)^{2}+\left(z+\frac{\nu v}{z}\right) v^{\prime}+\frac{2}{\beta-2} v+\alpha\left(\omega C_{3}\right)^{\beta-2} v^{\beta}=0, \quad v(1)=0 .
$$

Иных решений данного типа задача (4), (5) не имеет.

Доказательство. Пусть $z=\rho / a(t)$. Подставим выражение $u=\psi(t) v(z)$ в уравнение (4). После приведения подобных слагаемых и умножения обеих частей на $a^{2}(t) / \psi^{2}(t)$ получим

$$
v v^{\prime \prime}+\frac{1}{\sigma}\left(v^{\prime}\right)^{2}+\frac{a(t) a^{\prime}(t)}{\psi(t)} z v^{\prime}+\frac{\nu v v^{\prime}}{z}-\frac{a^{2}(t) \psi^{\prime}(t)}{\psi^{2}(t)} v+\alpha \frac{a^{2}(t) \psi^{\beta}(t)}{\psi^{2}(t)} v^{\beta}=0 .
$$

Так как функции $a$ и $\psi$ не зависят от $z$, условием превращения (10) в ОДУ является система

$$
\frac{a(t) a^{\prime}(t)}{\psi(t)}=A_{1}=\mathrm{const}, \quad \frac{a^{2}(t) \psi^{\prime}(t)}{\psi^{2}(t)}=A_{2}=\mathrm{const}, \quad \frac{a^{2}(t) \psi^{\beta}(t)}{\psi^{2}(t)}=A_{3}=\mathrm{const} .
$$

Система (11) является переопределенной. Найдем условия ее совместности.

Вначале обратим внимание на случай $A_{1}=0$. Он возможен тогда и только тогда, когда $a=$ const, $z=\rho / a$. Случай соответствует так называемой «остановившейся тепловой волне» [17, с. 68], однако поскольку нас в рамках данного исследования интересуют движущиеся тепловые волны, здесь он не рассматривается.

Пусть теперь $A_{1} \neq 0$. Тогда, выразив $\psi(t)$ через $a(t)$ из первого уравнения (11) и подставив во второе, получим

$$
\frac{\left(a^{\prime}\right)^{2}+a a^{\prime \prime}}{\left(a^{\prime}\right)^{2}}=\frac{A_{2}}{A_{1}^{2}}
$$

Отсюда следует

$$
\left(\frac{a}{a^{\prime}}\right)^{\prime}=2-\frac{A_{2}}{A_{1}^{2}}
$$

Примем для определенности $A_{1}=1$ (это можно сделать, не теряя общности рассмотрения). Возможны два случая.

1. Если $2-A_{2}=0$, то

$$
a(t)=C_{1} \exp \left(C_{2} t\right), \quad \psi(t)=a a^{\prime}=C_{1}^{2} C_{2} \exp \left(2 C_{2} t\right) .
$$

2. Если $2-A_{2}=1 / \omega \neq 0$, то

$$
a(t)=\left(C_{3} t+C_{4}\right)^{\omega}, \quad \psi(t)=a a^{\prime}=\omega C_{3}\left(C_{3} t+C_{4}\right)^{2 \omega-1} .
$$


Теперь рассмотрим третье уравнение (11). В первом случае, в котором функции $a(t)$ и $\psi(t)$ показательные, оно, очевидно, обращается в верное равенство тогда и только тогда, когда $\beta=1 ; A_{3}=1 / C_{2}$.

Во втором случае, где функции $a(t)$ и $\psi(t)$ степенные, оно обращается в верное равенство тогда и только тогда, когда $\omega=(\beta-2) /(2-2 \beta)$. Отсюда $\beta \neq 1 ; A_{3}=\left(\omega C_{3}\right)^{\beta-2}$. Кроме того, $\beta \neq 2$, поскольку иначе вновь получаем $a=$ const.

Можно видеть, что при подстановке полученных выражений для $a(t)$ и $\psi(t)$ в уравнение (10) первом случае получается уравнение (8), а во втором - (9). Таким образом, установлено, что в рассмотренных случаях задача (4), (5) действительно имеет обобщенно-автомодельные решения указанного в условии теоремы вида. С другой стороны, поскольку в ходе доказательства были рассмотрены все возможные случаи, иных подобных решений не существует.

Теорема 2. Задача (4), (5) имеет решения типа обобщенной бегущей волны в следующих случаях:

1) $\nu=0, \beta=2, \varphi_{1}(t, \rho)=\rho-\ln \left(C_{5} t+C_{6}\right)^{\omega}, \psi(t)=\omega C_{5} /\left(C_{5} t+C_{6}\right)$,

a $v(z)$ удовлетворяет задаче

$$
v v^{\prime \prime}+\frac{1}{\sigma}\left(v^{\prime}\right)^{2}+v^{\prime}+\frac{1}{\omega} v+\alpha v^{2}=0, \quad v(0)=0
$$

2) $\nu=0, \beta>0, \varphi_{1}(t, \rho)=\rho-\left(C_{7} t-C_{8}\right), \psi(t)=C_{7}$,

a $v(z)$ удовлетворяет задаче

$$
v v^{\prime \prime}+\frac{1}{\sigma}\left(v^{\prime}\right)^{2}+v^{\prime}+\alpha v^{\beta}=0, \quad v(0)=0 .
$$

Иных решений данного типа задача (4), (5) не имеет.

Доказательство. Пусть $z=\rho-a(t)$. Подставим выражение $u=\psi(t) v(z)$ в уравнение (4). После приведения подобных слагаемых и деления обеих частей на $\psi^{2}(t)$ получим

$$
v v^{\prime \prime}+\frac{1}{\sigma}\left(v^{\prime}\right)^{2}+\frac{a^{\prime}(t)}{\psi(t)} v^{\prime}-\frac{\psi^{\prime}(t)}{\psi^{2}(t)} v+\alpha \psi^{\beta-2}(t) v^{\beta}=0 .
$$

Поскольку функции $a$ и $\psi$ не зависят от $z$, условием превращения (14) в ОДУ является система

$$
\frac{a^{\prime}(t)}{\psi(t)}=B_{1}=\text { const }, \quad \frac{\psi^{\prime}(t)}{\psi^{2}(t)}=B_{2}=\text { const }, \quad \psi^{\beta-2}(t)=B_{3}=\text { const. }
$$


Первое уравнение системы (15) содержит только одну искомую функцию, поэтому проинтегрируем его отдельно.

Вначале рассмотрим случай $B_{1} \neq 0$. Вновь, не теряя общности рассмотрения, положим $B_{1}=1$. Тогда $\psi(t)=C_{5} /\left(C_{5} t+C_{6}\right)$. Подставив это выражение в первое уравнение $(15)$, получим, что $a(t)=\ln \left(C_{5} t+C_{6}\right)^{\omega}$, где $\omega=-1 / B_{2}$.

Пусть теперь $B_{1}=0$. Это возможно тогда и только тогда, когда $\psi=C_{7}$, где $C_{7}$ - произвольная константа. Тогда из второго уравнения (15) следует, что $a(t)=C_{7} t+C_{8}$.

Теперь рассмотрим третье уравнение (15). В первом случае оно обращается в верное равенство тогда и только тогда, когда $\beta=2$. Во втором случае оно всегда справедливо.

Можно видеть, что при подстановке полученных выражений для $a(t)$ и $\psi(t)$ в уравнение (14) в первом случае получается уравнение (12), а во втором - (13). Таким образом, установлено, что в рассмотренных случаях задача (4), (5) действительно имеет решения в виде обобщенной бегущей волны с логарифмическим и линейным фронтом. С другой стороны, поскольку в ходе доказательства рассмотрены все возможные случаи, иных подобных решений не существует.

Отметим важный частный случай рассматриваемой задачи. Пусть $\alpha=0$ (источника или стока нет). Тогда уравнение (4) имеет вид

$$
u_{t}=u\left(u_{\rho \rho}+\frac{\nu u_{\rho}}{\rho}\right)+\frac{u_{\rho}^{2}}{\sigma}
$$

и является классическим одномерным уравнением фильтрации [19] (или "the porous medium equation" [25]).

Справедливы нижеследующие утверждения.

Следствие 1. Задача (16), (5) имеет обобщенно-автомодельные решения в следующих случаях:

1) $\nu=0,1,2, \varphi_{2}(t, \rho)=\rho /\left[C_{1} \exp \left(C_{2} t\right)\right], \psi(t)=C_{1}^{2} C_{2} \exp \left(2 C_{2} t\right)$,

a $v(z)$ удовлетворяет задаче

$$
v v^{\prime \prime}+\frac{1}{\sigma}\left(v^{\prime}\right)^{2}+\left(z+\frac{\nu v}{z}\right) v^{\prime}-2 v=0, \quad v(1)=0
$$

2) $\nu=0,1,2, \varphi_{2}(t, \rho)=\rho /\left(C_{3} t+C_{4}\right)^{\omega}, \psi(t)=\omega C_{3}\left(C_{3} t+C_{4}\right)^{2 \omega-1}$,

a $v(z)$ удовлетворяет задаче

$$
v v^{\prime \prime}+\frac{1}{\sigma}\left(v^{\prime}\right)^{2}+\left(z+\frac{\nu v}{z}\right) v^{\prime}-\frac{2 \omega-1}{\omega} v=0, \quad v(1)=0 .
$$


Следствие 2. Задача (16), (5) имеет решения типа обобщенной бегущей волны в следующих случаях:

1) $\nu=0, \varphi_{1}(t, \rho)=\rho-\ln \left(C_{5} t+C_{6}\right)^{\omega}, \psi(t)=\omega C_{5} /\left(C_{5} t+C_{6}\right)$,

a $v(z)$ удовлетворяет задаче

$$
v v^{\prime \prime}+\frac{1}{\sigma}\left(v^{\prime}\right)^{2}+v^{\prime}+\frac{1}{\omega} v=0, \quad v(0)=0 ;
$$

2) $\nu=0, \varphi_{1}(t, \rho)=\rho-\left(C_{7} t-C_{8}\right), \psi(t)=C_{7}$, а $v(z)=-\sigma z$.

Справедливость данных утверждений вытекает из доказательств теорем 1 и 2 соответственно: за счет того, что $\alpha=0$, в системах (11) и (15) пропадает третье уравнение, что снимает дополнительные ограничения на параметры. Особого упоминания заслуживает п. 2 из следствия 2: в этом случае уравнение (13) интегрируется в квадратурах. В результате получается линейное решение уравнения (16) - простейшая бегущая волна.

Замечание 1. Решения (16), (17)-(19) представляют собой наиболее естественные виды решений задачи (16), (5), полученных ранее в [11]. Попытка использовать в качестве $z$ выражение $\varphi_{3}(t, \rho)=\rho / a_{1}(t)+a_{2}(t)$, которое обобщает (7), приводит к уже полученным в следствиях 1 и 2 решениям.

Замечание 2. Обыкновенные дифференциальные уравнения второго порядка, полученные в ходе доказательств теорем 1 и 2, иногда называют обобщенными уравнениями Льенара (см., например, [14]).

\section{§3. Теоремы существования и единственности аналитических решений}

Начальные задачи (8), (9), (12), (13) обладают весьма специфическими свойствами, которые они наследуют от исходной задачи (4), (5). В частности, для всех них задано нулевое начальное условие, которое приводит к тому, что в начальной точке ( $z=0$ либо $z=1)$ обращается в нуль множитель перед старшей производной, т. е. задачи не могут быть записаны в нормальной форме и для них не применимы классические теоремы существования решений задачи Коши. Вопросы существования и единственности решений в данном случае требуют специального исследования, которое и является содержанием настоящего параграфа.

Вначале рассмотрим задачи (8) и (9). Очевидно, что первая из них является частным случаем второй (при $\beta=1$ ).

Теорема 3. Пусть $\beta \in \mathbb{N}, \beta \neq 2$. Тогда у (9) существует единственное нетривиальное аналитическое решение в некоторой окрестности точки $z=1$. 
Доказательство. Под аналитическим мы понимаем решение, совпадающее в окрестности некоторой точки со своим тейлоровским разложением. Соответственно, решение (9) будем строить в виде степенного ряда

$$
v(z)=\sum_{n=0}^{+\infty} \frac{v_{n}(z-1)^{n}}{n !}, \quad v_{n}=\left.\frac{d^{n} v(z)}{d z^{n}}\right|_{z=1} .
$$

В данном случае $v_{0}=0$. Для того чтобы найти $v_{1}$, положим в уравнении (9) $v=0, z=1$. Получим квадратное уравнение, имеющее два корня:

1) $v_{1}=0$, который приводит к тривиальному решению $v \equiv 0$;

2) $v_{1}=-\sigma$, которое будем рассматривать далее.

Прочие коэффициенты ряда (20) при $n \geq 2$ однозначно определяются индукцией по $n$ из рекуррентных соотношений

$$
\begin{aligned}
v_{n+1}=\frac{1}{(\sigma n+1)}[ & \sum_{k=0}^{n-2} C_{n}^{k} v_{k+2} v_{n-k}+\frac{1}{\sigma} \sum_{k=1}^{n-1} C_{n}^{k} v_{k+1} v_{n-k+1} \\
& \left.+\frac{\nu}{2} \sum_{k=1}^{n} \frac{n !}{k !}(-1)^{n-k}\left(\sum_{i=1}^{k} C_{k+1}^{i} v_{i} v_{k+1-i}\right)+\left(n+\frac{2}{\beta-2}\right) v_{n}+\alpha F_{n}\right],
\end{aligned}
$$

где

$$
F_{n}=\left.\frac{d^{n}\left(v^{\beta}\right)}{d z^{n}}\right|_{z=1} .
$$

Выражение для $F_{n}$ здесь не приводится, так как в общем случае является громоздким. Принципиальным здесь является то, что при $\beta \in \mathbb{N}$ каждое $F_{n}$ определяется однозначно, причем зависит от коэффициентов $v_{k}$ при $k \leq n$.

Сходимость ряда (20) доказывается методом мажорант. Для построения мажорантной задачи перейдем к новой независимой переменной $s$ новой неизвестной функции $v_{*}(s)$ по формулам

$$
s=z-1, \quad v(z)=-\sigma s+s^{2} v_{*}(s) .
$$

Подставляя (21) в (9), приходим к уравнению

$$
\begin{aligned}
D_{1} v_{*} & +D_{2} s v_{*}^{\prime}+D_{3} s^{2} v_{*}^{\prime \prime} \\
& =D_{4}+s g_{1}\left(z, v_{*}\right)+s^{2} g_{2}\left(s, v_{*}, v_{*}^{\prime}\right)+s^{3} g_{3}\left(s, v_{*}, v_{*}^{\prime}, v_{*}^{\prime \prime}\right),
\end{aligned}
$$

где $D_{1}, D_{2}, D_{3}>0, D_{4} \in \mathbb{R}$, а $g_{1}, g_{2}, g_{3}$ являются аналитическими функциями своих аргументов, их конкретный вид для доказательства несуществен. Из $(22)$ можно получить значения $v_{*}(0)$ и $v_{*}^{\prime}(0)$. 
Мажорантная задача для уравнения (22) имеет вид

$$
V^{\prime \prime}=E\left(\frac{\partial G_{1}}{\partial s}+\frac{\partial G_{1}}{\partial V} V^{\prime}+G_{2}+s G_{3}\right), \quad V(0)=V_{0}, \quad V^{\prime}(0)=V_{1},
$$

где

$$
\begin{gathered}
E=\sup _{n}\left\{\frac{(n-1) n+1}{D_{1}+n D_{2}+(n-1) n D_{3}}\right\}, \quad V_{0}>\left|v_{*}(0)\right|, \quad V_{1}>\left|v_{*}^{\prime}(0)\right|, \\
G_{1}=G_{1}(s, V), \quad G_{2}=G_{2}\left(s, V, V^{\prime}\right), \quad G_{3}=G_{3}\left(s, V, V^{\prime}, V^{\prime \prime}\right), \quad G_{i} \gg g_{i}, \quad i=\overline{1,3} .
\end{gathered}
$$

Докажем, что у задачи (23) имеется аналитическое мажорирующее нуль решение. Положим $s=0$ и подставим в правую часть уравнения (23) $V_{0}, V_{1}$; тогда $V_{2}=V^{\prime \prime}(0)$ выражается однозначно, причем $V_{2}>0$.

Дифференцируя (23) по $s$, а затем выражая $V^{\prime \prime \prime}$, приходим к дифференциальному уравнению третьего порядка с аналитической мажорирующей нуль правой частью:

$$
\begin{aligned}
V^{\prime \prime \prime}=\frac{E}{1-E s\left(G_{3}\right)_{V^{\prime \prime}}}[ & \left(G_{1}\right)_{s V}+2\left(G_{1}\right)_{s V V} V^{\prime}+\left(G_{1}\right)_{s s V V} V_{s}+\left(G_{1}\right)_{V V} V_{s s} \\
& +\left(G_{2}\right)_{s}+\left(G_{2}\right)_{V} V^{\prime}+\left(G_{2}\right)_{V^{\prime}} V^{\prime \prime} \\
& \left.+\left(G_{3}\right)+s\left(G_{3}\right)_{s}+s\left(G_{3}\right)_{V} V^{\prime}+s\left(G_{3}\right)_{V^{\prime}} V^{\prime \prime}\right] .
\end{aligned}
$$

Величины $V_{0}, V_{1}, V_{2}$ - начальные условия для уравнения (24) - являются строго положительными; значит, по теореме Коши о существовании аналитического решения, в некоторой окрестности точки $s=0$ у задачи (23) существует единственное решение - аналитическая мажорирующая нуль функция $V(s)$. Следовательно, функции $v_{*}(z)$ и $v(z)$ также являются аналитическими.

Уравнения (12), (13) могут быть записаны совместно в виде

$$
v v^{\prime \prime}+\frac{1}{\sigma}\left(v^{\prime}\right)^{2}+v^{\prime}+\kappa v+\alpha v^{2}=0
$$

где $\kappa=1 / \omega$ в случае (12), либо $\kappa=0$ в случае (13).

Теорема 4. Пусть $v(0)=0, v^{\prime}(0)=-\sigma$. Тогда у (25) существует единственное нетривиальное аналитическое решение в некоторой окрестности точки $z=0$.

Справедливость данного утверждения следует из ранее доказанной теоремы в [10]. 


\section{§4. Исследование особых точек}

Начиная с этого параграфа, рассмотрим более подробно случай обобщенной бегущей волны. Для этого сначала выполним качественное исследование уравнения (25). Поскольку оно является автономным, перейдем к рассмотрению фазовой плоскости $\left(v, v^{\prime}=w\right)$. Будем использовать технику, предложенную в [2] (см. также [11]).

Уравнению (25) соответствует динамическая система

$$
\frac{d v}{d z}=w, \quad \frac{d w}{d z}=-\frac{w^{2} / \sigma+w+\alpha v^{2}+\kappa v}{v} .
$$

Изменим в системе (26) параметризацию, полагая $d z=v d \zeta$. В результате получим

$$
\frac{d v}{d \zeta}=v w, \quad \frac{d w}{d \zeta}=-\frac{w^{2}}{\sigma}-w-\alpha v^{2}-\kappa v,
$$

доопределив тем самым (26) в точках $v=0$ по непрерывности.

Всюду, где $v \neq 0$, траектории систем (26) и (27) совпадают, хотя параметры на них различны. При $v>0$ направления по $\zeta$ и $z$ совпадают, а при $v<0$ они противоположны. Точки, в которых $v=0$, не принадлежат траекториям системы (26). Таким образом, мы перешли от уравнения (25) к динамической системе (27). Исследуем теперь состояния равновесия (особые точки) системы (27).

Возможны следующие случаи:

$1)$ если $\kappa \neq 0$, то имеется три состояния равновесия: $(0,-\sigma),(0,0)$ и $(-\kappa / \alpha, 0)$;

$2)$ если $\kappa=0$, то имеется два состояния равновесия: $(0,-\sigma)$ и $(0,0)$.

Введем следующие обозначения:

$$
\begin{gathered}
P(v, w)=v w, \quad Q(v, w)=-w^{2} / \sigma-w-\alpha v^{2}-\kappa v \\
M(v, w)=\left(\begin{array}{ll}
P_{v} & P_{w} \\
Q_{v} & Q_{w}
\end{array}\right)=\left(\begin{array}{cc}
w & v \\
-2 \alpha v-\kappa & -2 w / \sigma-1
\end{array}\right), \\
\Delta(v, w)=\operatorname{det} M(v, w)=-\frac{2 w^{2}}{\sigma}-w+2 \alpha v^{2}+\kappa v, \\
\delta(v, w)=\operatorname{Tr} M(v, w)=\frac{(\sigma-2) w}{\sigma}-1 .
\end{gathered}
$$

Определим последовательно тип каждой особой точки.

1. Рассмотрим точку $(0,-\sigma)$. Поскольку $\Delta(0,-\sigma)=-\sigma \neq 0$, то это простое состояние равновесия;

$$
\left.\operatorname{det}(M-\lambda E)\right|_{v=0, w=-\sigma}=(\lambda+\sigma)(\lambda-1),
$$


т. е. корнями характеристического уравнения являются $\lambda_{1}=-\sigma$ и $\lambda_{2}=1$. Так как здесь $\Delta<0, \lambda_{1}, \lambda_{2} \in \mathbb{R}$ и $\lambda_{1} \lambda_{2}<0$, точка $(0,-\sigma)$ имеет топологический тип седло.

2. Рассмотрим точку $(0,0)$. Поскольку $\Delta(0,0)=0$, то это сложное состояние равновесия. Здесь $\delta(0,0)=-1 \neq 0$, уравнение, которое получается из системы (27) при исключении независимой переменной $\zeta$, представимо в виде

$$
v w d w-\left[l w-w^{2} / \sigma-\alpha v^{2}-\kappa v\right] d v=0,
$$

где $l=-1$. Далее отдельно рассмотрим случаи $\kappa=1 / \omega$ и $\kappa=0$.

2.1. Пусть $\kappa=1 / \omega \neq 0$. Представим решение уравнения

$$
-l w+w^{2} / \sigma+\alpha v^{2}+\kappa v=0
$$

в виде ряда по степеням $v$, который подставим в $v w$. Получим

$$
w=\phi(v)=-v / \omega+\ldots, \quad \xi(v)=\left.(v w)\right|_{w=\phi(v)}=-v^{2} / \omega+\ldots .
$$

Поскольку младшая степень $v$ в разложении $\xi(v)$ равна двум, точка $(0,0)$ является седло-узлом с одним узловым сектором и двумя седловыми; так как $l<0$, узловой сектор устойчивый. При этом если $\omega<0$, то траектории узлового сектора стремятся к $(0,0)$ при $\zeta \rightarrow-\infty$ слева от оси $O w$, а если $\omega>0$, то справа от оси $O w$ при $\zeta \rightarrow+\infty$.

2.2. Пусть $\kappa=0$. Тогда разложения из п. 2.1 примут вид

$$
w=\phi(v)=-\frac{\alpha}{2} v^{2}+\ldots, \quad \xi(v)=-\frac{\alpha}{2} v^{3}+\ldots .
$$

Младшая степень $v$ в разложении $\xi(v)$ равна трем, поэтому при $\alpha>0$ точка $(0,0)$ является сложным узлом (устойчивым), а при $\alpha<0-$ сложным седлом.

3. Рассмотрим точку $(-\kappa / \alpha, 0)$. Поскольку

$$
\Delta(-\kappa / \alpha, 0)=\kappa^{2} / \alpha \neq 0,
$$

то это простое состояние равновесия;

$$
\left.\operatorname{det}(M-\lambda E)\right|_{v=-\kappa / \alpha, w=0}=\lambda^{2}+\lambda+\kappa^{2} / \alpha,
$$

т. е. корнями характеристического уравнения будут

$$
\lambda_{1,2}=-1 / 2 \pm \sqrt{1 / 4-\kappa^{2} / \alpha}=-1 / 2 \pm \sqrt{1 / 4-1 /\left(\alpha \omega^{2}\right)} .
$$

В зависимости от значений параметров $\alpha$ и $\omega$ рассматриваемое состояние равновесия может являться либо устойчивым фокусом, если $1 / 4<$ $1 /\left(\alpha \omega^{2}\right)$, либо устойчивым узлом, если $1 / 4>1 /\left(\alpha \omega^{2}\right)$. Случай $1 / 4=1 /\left(\alpha \omega^{2}\right)$ требует особого рассмотрения, которое здесь не проводится. 


\section{§5. Исследование фазового портрета}

Построим и исследуем фазовый портрет системы (27) в случае, когда $\alpha>0$. Не теряя общности рассмотрения, можем принять $\omega>0$, поскольку решения (25) обладают симметрией $v(z,-\omega)=-v(-z, \omega)$. Рассмотрим сначала тепловую волну с логарифмическим фронтом, а затем с линейным. Отметим, чтобы не повторяться, что во всех рассмотренных случаях

1) фазовые траектории меняют направление движения при переходе через ось $O v$, а также при пересечении эллипса $w^{2} / \sigma+w+\alpha v^{2}+\kappa v=0$, на котором, в частности, лежат все особые точки (он изображен пунктирной линией);

2) особые точки, лежащие на оси $O w$, обладают вертикальными полусепаратрисами.

5.1. Три особых точки, случай фокуса. На рис. 1 представлен фазовый портрет системы $(27)$ в случае, когда $1 / 4<1 /\left(\alpha \omega^{2}\right)$. Система в данном случае, как показано выше, имеет три особых точки: $M_{0}(0,0), M_{1}(0,-\sigma)$ и $M_{2}(-\kappa / \alpha, 0)$, представляющие собой седло-узел (с одним узловым и двумя седловыми секторами), седло и устойчивый фокус соответственно.

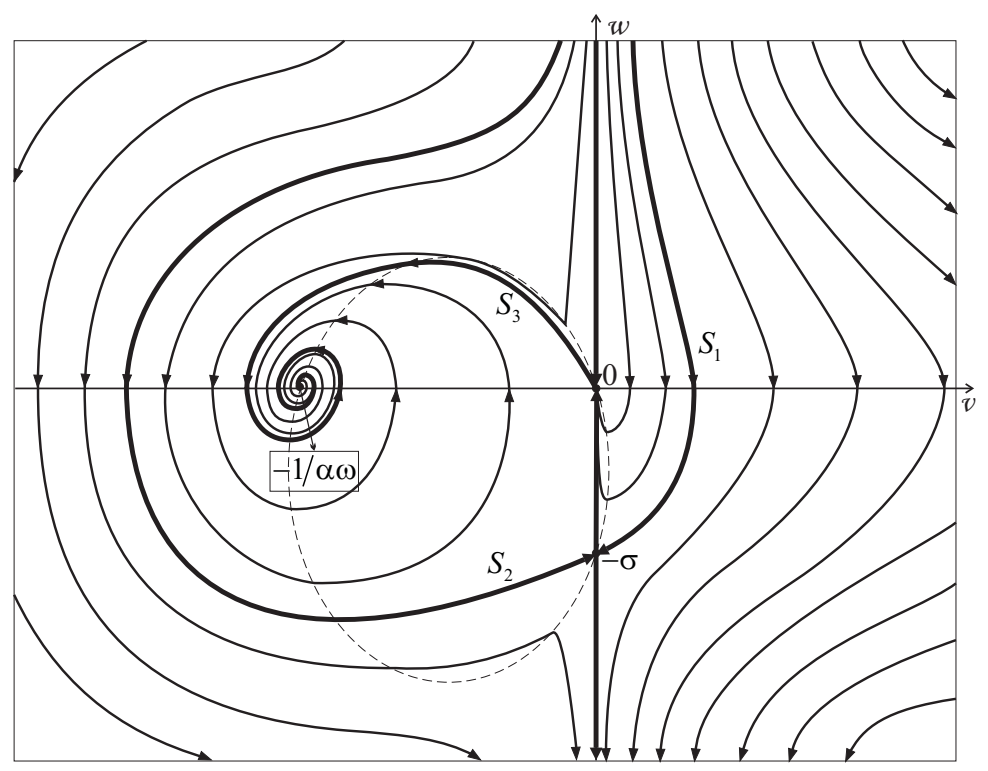

Рис. 1. Фазовый портрет системы (27) в случае, когда $M_{2}-$ фокус

Помимо сепаратрис, лежащих на оси $O w$, имеются еще три, которые не совпадают с координатными осями. Из них $S_{1}$ и $S_{2}$ стремятся к точке $M_{1}$ при $\zeta \rightarrow-0$ с положительным наклоном; $S_{3}$ стремится

a) к точке $M_{0}$ при $\zeta \rightarrow+0$ с отрицательным наклоном;

b) к точке $M_{2}$ при $\zeta \rightarrow+\infty$. 
Сепаратриса $S_{1}$ отделяет узловой сектор особой точки $M_{0}, S_{3}$ разделяет седловые сектора той же точки, $S_{2}$ окружает область закрутки фокуса.

Выполнив обратную параметризацию, т. е. вернувшись к системе (26), можно убедиться, что сепаратрисами $S_{1}^{*}, S_{2}^{*}$ и $S_{3}^{*}$, в которые в этом случае перейдут $S_{1}, S_{2}$ и $S_{3}$, исчерпывается набор траекторий, стремящихся при $z \rightarrow 0$ к точкам $(0,-\sigma)$ и $(0,0)$, следовательно, они отвечают решениям уравнения (25) с соответствующими начальными условиями.

Таким образом, по виду фазовых траекторий $S_{1}, S_{2}$ и $S_{3}$ можно описать поведение решений задачи Коши (зK) для (25): $S_{1} \cup S_{2}$ соответствует решению при

$$
v(0)=0, \quad v^{\prime}(0)=-\sigma
$$

(см. рис. $2(\mathrm{a}))$; решение зK (25), (28) существует на некотором интервале $\left(-z_{*}, z^{*}\right)$ и не может быть продолжено за его границы, при этом

$$
\left.v\right|_{z=z^{*}}=\left.v\right|_{z=-z_{*}}=0, \quad \lim _{z \rightarrow-z_{*}+0} v^{\prime}(z)=+\infty, \quad \lim _{z \rightarrow-z^{*}-0} v^{\prime}(z)=-\infty .
$$

Отметим, что на графике (рис. 2 (а)) имеется точка перегиба $z_{e}>0$, слева от которой график является выпуклым вверх $\left(v^{\prime \prime}<0\right)$, а справа - выпуклым вниз $\left(v^{\prime \prime}>0\right)$.
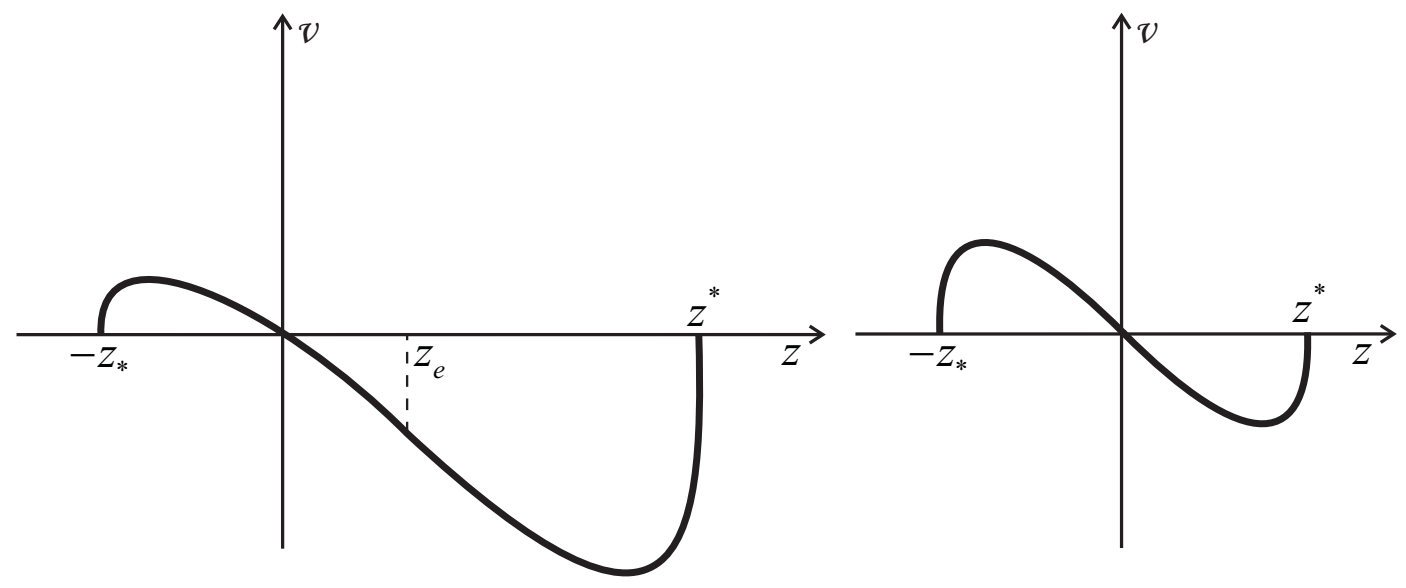

Рис. 2. График решения задачи Коши (25), (28):

(a) при $\omega \alpha>0$; (b) при $\kappa=0$

Сепаратриса $S_{3}$ соответствует нетривиальному решению при

$$
v(0)=0, \quad v^{\prime}(0)=0
$$

(см. рис. $3(\mathrm{a}))$; решение зK $(25)$, (30) на интервале $\left(-z_{\alpha}, 0\right)$ монотонно возрастает, а далее при $z<-z_{\alpha}, z \rightarrow-\infty$ немонотонно приближается к горизонтальной асимптоте $v=-1 /(\alpha \omega)$. 

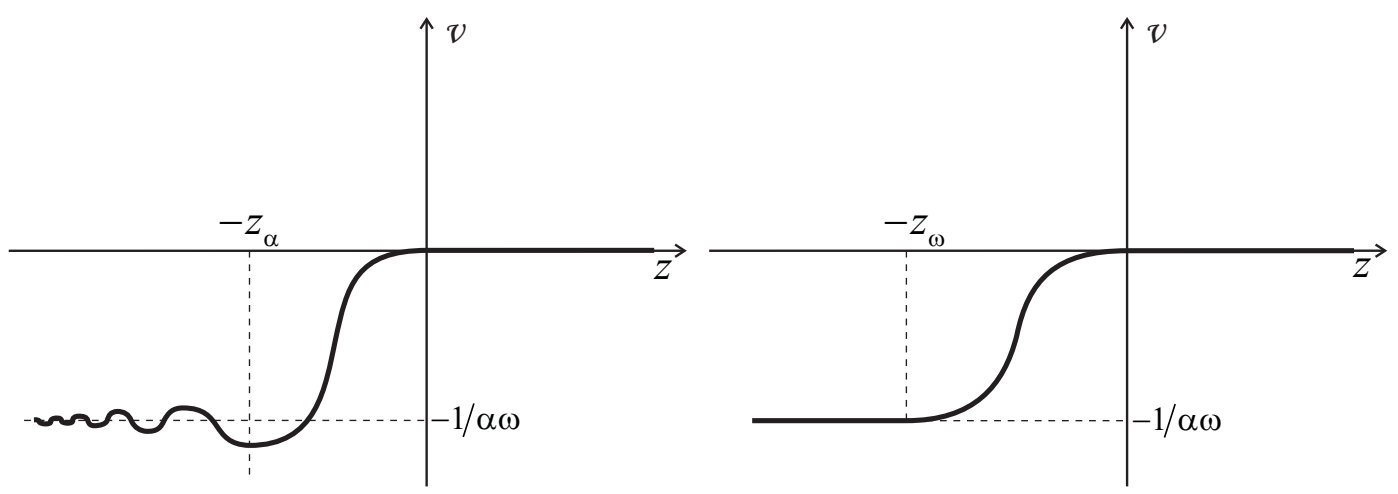

Рис. 3. График решения задачи Коши (25), (30):

(a) при $0<\omega^{2} \alpha<4$; (b) при $\omega^{2} \alpha>4$

5.2. Три особых точки, случай узла. На рис. 4 представлен фазовый портрет системы $(27)$ в случае, когда $1 / 4>1 /\left(\alpha \omega^{2}\right)>0$. Система также имеет три особых точки: $M_{0}(0,0), M_{1}(0,-\sigma)$ и $M_{2}(-\kappa / \alpha, 0)$, представляющие собой седло-узел, седло и устойчивый узел соответственно.

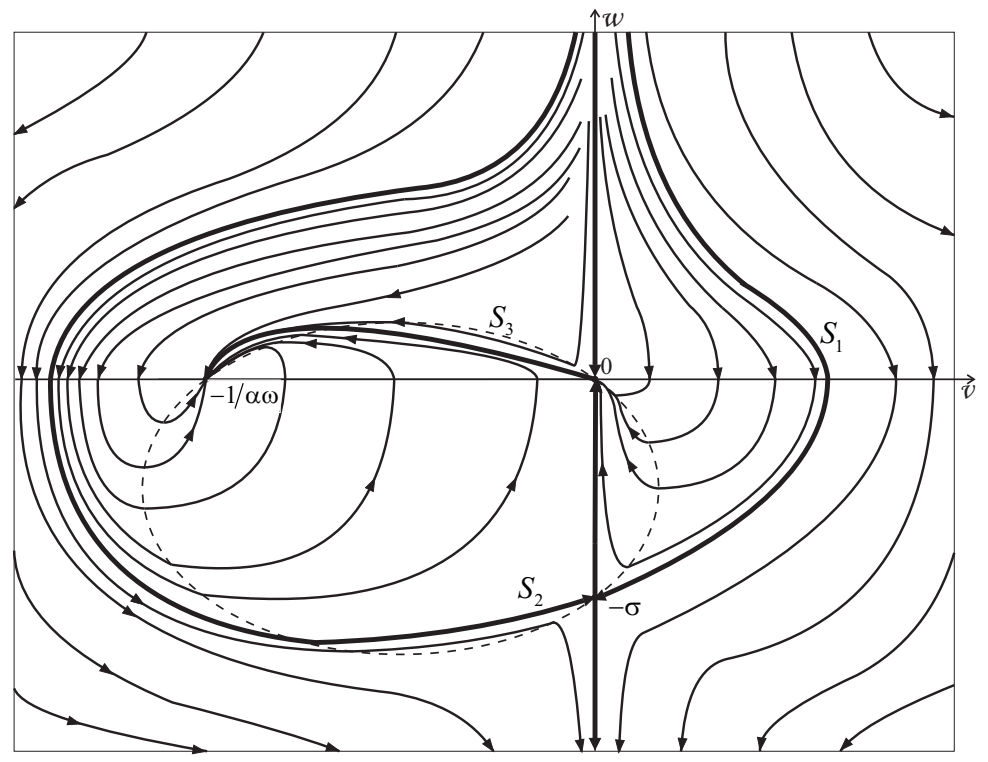

Рис. 4. Фазовый портрет системы $(27)$ в случае, когда $M_{2}-$ узел

Помимо сепаратрис, лежащих на оси $O w$, имеются еще три, которые не совпадают с координатными осями. Из них $S_{1}$ и $S_{2}$ стремятся к точке $M_{1}$ при $\zeta \rightarrow-0$ с положительным наклоном; $S_{3}$ стремится

a) к точке $M_{0}$ при $\zeta \rightarrow+0$ с отрицательным наклоном;

b) к точке $M_{2}$ при $\zeta \rightarrow+\infty$.

Сепаратриса $S_{1}$ отделяет узловой сектор особой точки $M_{0}, S_{3}$ разделяет седловые сектора той же точки (закрутки при этом, в отличие от предыдущего случая, не будет), $S_{2}$ окружает область притяжения особой точки $M_{1}$. 
Аналогично предыдущему случаю устанавливаем, что сепаратрисы $S_{1}$, $S_{2}$ и $S_{3}$ отвечают решениям уравнения (25) с соответствующими начальными условиями: $S_{1} \cup S_{2}$ - решению зК (25), (28), при этом последнее существует на некотором интервале $\left(-z_{*}, z^{*}\right)$ и не может быть продолжено за его пределы (качественное поведение данной кривой описывает рис. 2 (а), предельные свойства (29) сохраняются); сепаратриса $S_{3}$ соответствует нетривиальному решению зК (25), (30) (см. рис. 3 (b)), при этом решение на интервале $\left(-z_{\omega}, 0\right)$ монотонно возрастает, а далее при $z>-z_{\omega}$ может быть продолжено прямой $v=-1 /(\alpha \omega)$ (с непрерывными производными).

5.3. Две особых точки. На рис. 5 представлен фазовый портрет системы $(27)$ в случае, когда $\kappa=0$. Система имеет две особых точки: $M_{0}(0,0)$ и $M_{1}(0,-\sigma)$, представляющие собой устойчивый узел и седло соответственHо.

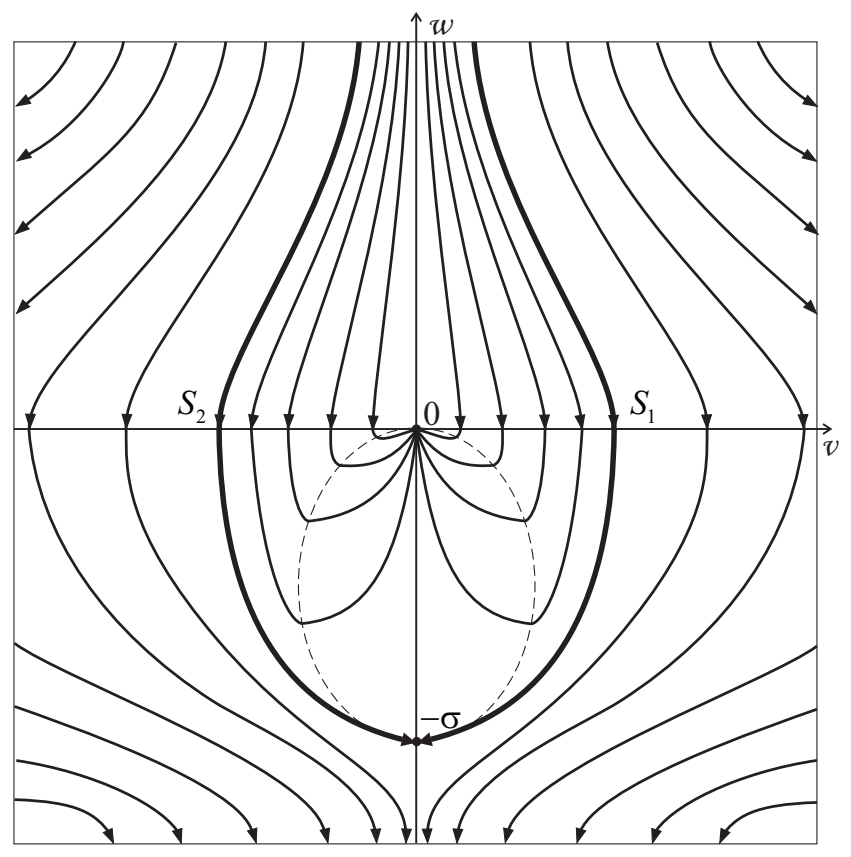

Рис. 5. Фазовый портрет системы (27) в случае, когда точки $M_{2}$ и $M_{0}$ совпадают

Помимо сепаратрис, лежащих на оси $O w$, имеются еще две, которые не совпадают с координатными осями. При этом $S_{1}$ и $S_{2}$ стремятся к точке $M_{1}$ при $\zeta \rightarrow-0$ с положительным наклоном и отделяют область притяжения узловой особой точки $M_{0} ; S_{1} \cup S 2$ соответствует решению зК (25), (28), причем последнее существует на некотором симметричном интервале $\left(-z_{*}, z_{*}\right)$ и не может быть продолжено за его пределы (см. рис. $\left.2(б)\right)$, $v(z)=-v(-z)$; более того, $\left.v\right|_{z= \pm z_{*}}=0, \lim _{z \rightarrow \pm z_{*} \mp 0} v^{\prime}(z)=\mp \infty$. Точкой переги- 
ба здесь является $z_{e}=0 . \mathrm{K}$ особой точке $M_{0}$ стремятся только полусепаратрисы, совпадающие с осью $O w$, поэтому зК (25), (30) в данном случае имеет только тривиальное решение.

Из рассуждений, приведенных в настоящем разделе, вытекает справедливость следующего утверждения.

Теорема 5. 1. Задача Коши (25), (28) в случае $\alpha>0$ имеет единственное решение $v(z) \in C_{\left[-z_{*}, z^{*}\right)} \cap C_{\left(-z_{*}, z^{*}\right)}^{2}$.

2. Задача Коши (25), (30) в случае $\alpha>0, \kappa>0$ имеет единственное нетривиальное решение $v(z) \in C_{(-\infty, 0]} \cap C_{(-\infty, 0)}^{2}$.

\section{§6. Поведение тепловых волн}

С использованием полученных ранее результатов можно более детально исследовать поведение решений типа обобщенной бегущей волны. Как и в предыдущем разделе, полагаем, что $\alpha>0$.

6.1. Тепловая волна с логарифмическим фронтом. Пусть для определенности фронт тепловой волны движется из начала координат в первый квадрант, т. е. для функции, определяющей закон его движения, справедливы соотношения $a(t)=\ln \left(c_{5} t+1\right)^{\omega}, \omega c_{5}>0$. Тогда по теореме 2 имеем

$$
u(t, \rho)=\frac{\omega c_{5} v(z)}{c_{5} t+1}
$$

где $z=\rho-\omega \ln \left(c_{5} t+1\right)$. Так как, не теряя общности рассмотрения, можно принять $\omega>0$, возможны два случая.

1. Пусть $c_{5}>0$. Очевидно, что в данном случае $u(t, \rho) \geq 0$ тогда и только тогда, когда $v(z) \geq 0$, поэтому рассмотрим неотрицательную часть решения задачи $(25),(28)$ (см. рис. $2(\mathrm{a}))$. Из результатов предыдущего параграфа известно, что в данном случае область распространения тепловой волны в любой момент времени определяется неравенствами

$$
\ln \left(c_{5} t+1\right)^{\omega}-z_{*} \leq \rho \leq \ln \left(c_{5} t+1\right)^{\omega}
$$

т. е. имеем уединенную волну с задним фронтом $\rho=\ln \left(c_{5} t+1\right)^{\omega}-z_{*}$. При этом из (31) следует, что $\lim _{t \rightarrow+\infty} u(t, \rho)=0$ для всех $\rho$ в силу ограниченности $v(\xi)$ на $\left[-z_{*}, 0\right]$, т. е. полное остывание нагретого полупространства происходит за бесконечное время. Геометрическая иллюстрация приведена на рис. 6 (а).

2. Пусть $c_{5}<0$. Тогда $0 \leq t<-1 / c_{5}$, причем $t=-1 / c_{5}-$ вертикальная асимптота фронта тепловой волны. Очевидно, что в данном случае $u(t, \rho) \geq 0$ тогда и только тогда, когда $v(\xi) \leq 0$, поэтому возможны следующие подслучаи. 

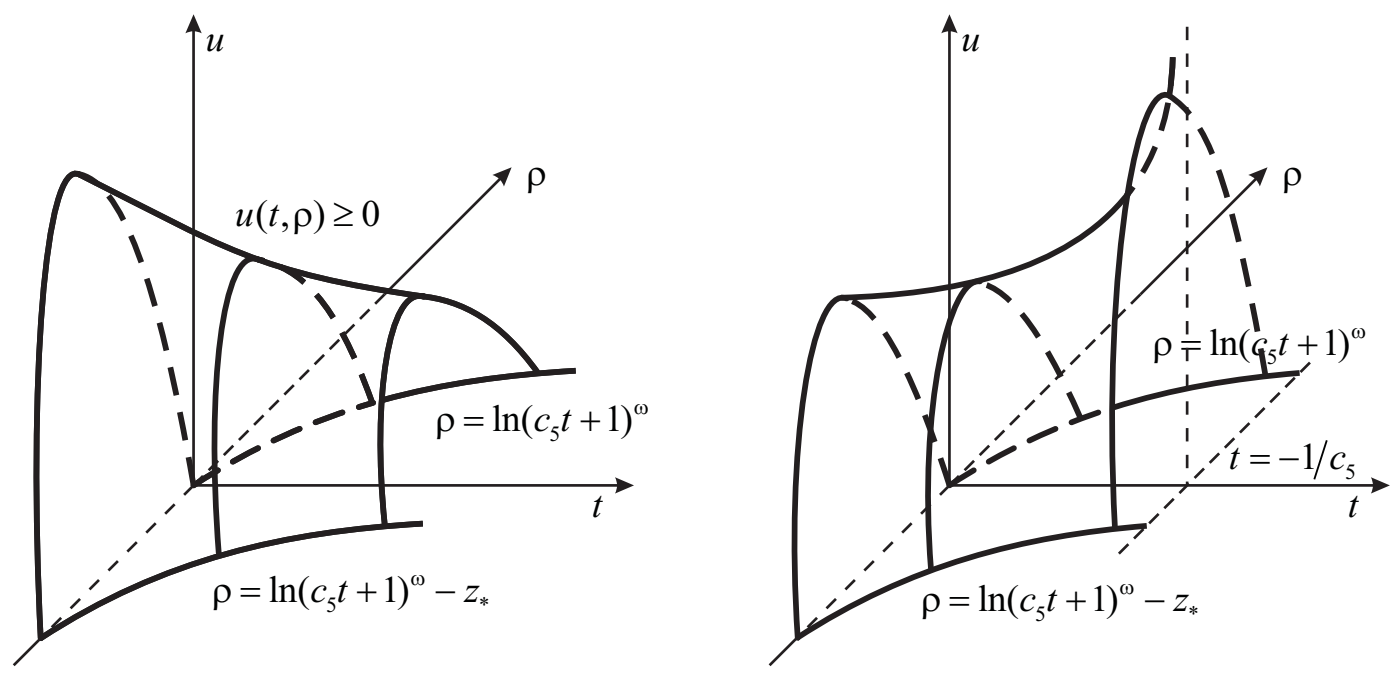

Рис. 6. Тепловая волна с логарифмическим фронтом:

(a) затухание; (b) обострение

2.1. Пусть $v(z) \leq 0, v^{\prime}(0)=-\sigma$ (см. рис. $\left.2(\mathrm{a})\right)$. Из результатов предыдущего параграфа следует, что область, в которой задана тепловая волна, определяется неравенством

$$
\ln \left(c_{5} t+1\right)^{\omega}-z^{*} \leq \rho \leq \ln \left(c_{5} t+1\right)^{\omega}
$$

т. е. вновь имеем уединенную волну с задним фронтом $\rho=\ln \left(c_{5} t+1\right)^{\omega}-z_{*}$, форма и поведение которой отличаются от предыдущего случая. В частности, бесконечно растет амплитуда, причем нагрев до сколь угодно большой температуры происходит за конечное время. Такое поведение соответствует HS-режиму с обострением [17, с. 69]. Геометрическая иллюстрация приведена на рис. 6 (b).

2.2. Пусть $v(z) \leq 0, v^{\prime}(0)=0$. Рассмотрим нетривиальное решение зK (25), (30) (см. рис. 3). Поскольку здесь $z \leq 0$, из результатов предыдущего параграфа следует, что область распространения тепловой волны определяется неравенством $\rho \geq \ln \left(c_{1} t+1\right)^{\omega}$. Такая тепловая волна, очевидно, не может быть порождена каким-либо краевым режимом при $\rho=0$, однако построенное решение, по-видимому, может быть полезно при исследовании обратных задач теплопроводности [13].

Замечание 3. Наиболее интересным из рассмотренных в данном пункте случаев представляется случай 2.1, поскольку здесь поведение тепловой волны принципиально отличается от рассмотренного ранее случая, когда источник отсутствует: как явствует из результатов работы [11], при $\alpha=0$ в режиме с обострением не происходит локализации теплового воздействия. 
6.2. Тепловая волна с линейным фронтом. Пусть фронт тепловой волны движется из начала координат в первый квадрант, т. е. для функции, определяющей закон его движения, справедливы соотношения $a(t)=$ $c_{7} t, c_{7}>0$. Тогда по теореме 2 имеем

$$
u(t, \rho)=c_{7} v(z),
$$

где $z=\rho-c_{7} t$. Так как в данном случае $u(t, \rho) \geq 0$ тогда и только тогда, когда $v(z) \geq 0$, рассмотрим неотрицательную часть решения задачи $(25)$, (28) при $\kappa=0$ (см. рис. $2(\mathrm{a}))$. Из результатов предыдущего параграфа известно, что в данном случае область распространения тепловой волны в любой момент времени определяется неравенствами $c_{7} t-z_{*} \leq \rho \leq c_{7} t$, т. е. имеем уединенную волну с задним фронтом $\rho=c_{7} t-z_{*}$. При этом из (32) следует, что данная волна движется, не меняя своей формы, иначе говоря, является солитоном в классическом понимании этого термина. Геометрическая иллюстрация приведена на рис. 7.

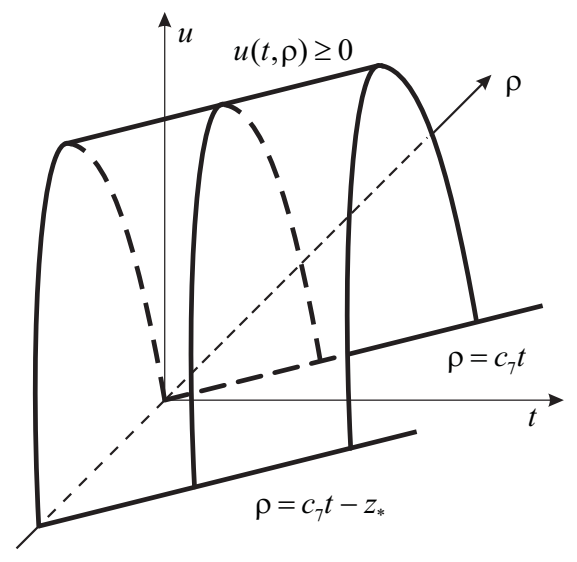

Рис. 7. Тепловая волна с линейным фронтом - солитон

Замечание 4. Здесь поведение тепловой волны также принципиально отличается от рассмотренного ранее случая, когда источник отсутствует: при $\alpha=0$ имеет место простейшее линейное решение задачи (4), (5) (см. следствие 2). Таким образом, в обоих случаях (с источником и без него) функция $u=u(t, \rho)$ определяет линейчатую поверхность в пространстве переменных $t, \rho, u$, однако в первом из них решение, очевидно, является более содержательным.

\section{Заключение}

В настоящей работе получены новые классы точных (обобщенно-автомодельных и типа обобщенной бегущей волны) решений нелинейного 
уравнения теплопроводности в случае степенной зависимости коэффициента теплопроводности от температуры при наличии источника (стока) со степенной зависимостью последнего от температуры. Найденные решения имеют вид тепловой волны, распространяющейся по холодному (нулевому) фону с конечной скоростью. Определены семейства допустимых фронтов, являющихся логарифмическими, степенными и показательными функциями. Исходная задача построения решений нелинейного параболического уравнения второго порядка с частными производными сведена к обыкновенным дифференциальным уравнениям второго порядка с начальными условиями, предполагающими вырождение уравнений. Обоснованы теоремы существования и единственности локально-аналитических решений указанных начальных задач.

Проведено специальное исследование решений, имеющих тип обобщенной бегущей волны (с логарифмическим и линейным фронтом), при наличии источника. Методами качественной теории дифференциальных уравнений установлена нелокальная разрешимость соответствующих задач Коши в классе функций конечной гладкости, изучены поведение и глобальные свойства решений.

Полученные результаты интерпретированы с точки зрения исходной задачи о движении тепловой волны. Показано, что в случае логарифмического теплового фронта решение имеет вид уединенной волны, амплитуда которой (в зависимости от выбора направления движения) может либо затухать, стремясь к нулю при $t \rightarrow+\infty$, либо возрастать, стремясь к бесконечности при $t \rightarrow$ const (HS-режим с обострением). В случае линейного теплового фронта решение имеет вид солитона, движущегося по холодному фону с постоянной скоростью.

Дальнейшие исследования в данном направлении могут быть следующими. Во-первых, интерес представляет получение оценок радиуса сходимости построенных степенных рядов. Во-вторых, целесообразно выполнить качественное исследование обыкновенных дифференциальных уравнений, к которым сводится построение обобщенно-автомодельных решений.

\section{Список литературы}

1. Баренблатт Г. И., Ентов В. М., Рыжик В. М. Теория нестационарной фильтрачии жидкости и газа. М.: Недра, 1972.

2. Баутин Н. Н., Леонтович Е. А. Методъ и приемы качественного исследования динамических систем на плоскости. М.: Наука, 1990.

3. Врагов В. Н. Краевые задачи для неклассических уравнений математической физики. Новосибирск: Изд-во НГУ, 1983. 
4. Демиденко Г. В. Квазиэллиптические операторы и уравнения соболевского типа // Сиб. матем. журн. 2008. Т. 49, № 5. С. 1064-1076.

5. Зельдович Я. Б., Компанеец А. С. К теории распространения тепла при теплопроводности, зависящей от температуры // Сборник, посвященныц 70-летию А. Ф. Иоффе. М.: Изд-во АН СССР, 1950. С. 61-71.

6. Казаков А. Л., Кузнецов П. А. Об одной краевой задаче для нелинейного уравнения теплопроводности в случае двух пространственных переменных // Сиб. журн. индустр. матем. 2014. Т. 17, № 1(57). C. $46-54$.

7. Казаков А. Л., Кузнецов П. А. Об аналитических решениях одной специальной краевой задачи для нелинейного уравнения теплопроводности в полярных координатах // Сиб. журн. индустр. матем. 2018. Т. 21, № 2(74). С. 56-65.

8. Казаков А. Л., Кузнецов П. А., Спевак Л. Ф. Об одной краевой задаче с вырождением для нелинейного уравнения теплопроводности в сферических координатах // Тр. ИММ УрО РАН. 2014. Т. 20, №1. С. 119 129.

9. Казаков А. Л., Лемперт А. А. О существовании и единственности краевой задачи для параболического уравнения нестационарной фильтрации // Прикл. мех. и техн. физ. 2013. Т. 54, № 2. С. 97-105.

10. Казаков А. Л., Орлов Св. С. О некоторых точных решениях нелинейного уравнения теплопроводности // Тр. ИММ УрО РАН. 2016. Т. 22, № 1. C. $112-123$.

11. Казаков А. Л., Орлов Св. С., Орлов С. С. Построение и исследование некоторых точных решений нелинейного уравнения теплопроводности // Сиб. матем. журн. 2018. Т. 59, №3. С. 544-560.

12. Кожанов А. И., Пинигина Н. Р. Краевые задачи для некоторых классов уравнений составного типа высокого порядка // Сиб. электрон. матем. изв. 2015. Т. 12. С. 842-853.

13. Короткий А. И., Стародубцева Ю. В. Моделирование прямых и обратных граничных задач для стационарных моделей тепломассопереноса. Екатеринбург: Изд-во Урал. ун-та, 2015.

14. Кудряшов Н. А., Синельщиков Д. И. Аналитические решения нелинейного уравнения конвекции-диффузии с нелинейными источниками // Моделир. и анал. инф. систем. 2016. Т. 23, № 3. С. 309-316.

15. Маслов В. П., Данилов В. Г., Волосов К. А. Математическое моделирование прочессов тепломассопереноса. Эволющия диссипативных структур. М.: Наука, 1987. 
16. Полянин А. Д., Зайцев В. Ф., Журов А. И. Нелинейнъе уравнения математической физики и механики. Методь решения. М.: Изд-во Юрайт, 2017.

17. Самарский А. А., Галактионов В. А., Курдюмов С. П., Михайлов А. П. Режимы с обострением в задачах для квазилинейных параболических уравнений. М.: Наука, 1987.

18. Свешников А. Г., Альшин А. Б., Корпусов М. О., Плетнер Ю. Д. Линейные и нелинейные уравнения соболевского типа. М.: Физматлит, 2007.

19. Сидоров А. Ф. Избраннъе труды: Математика. Механика. М.: Физматлит, 2001.

20. Demidenko G. V. Quasielliptic operators and equations not solvable with respect to the higher order derivative $/ / \mathrm{J}$. Math. Sci. (United States). 2018. T. 230, № 1. P. 25-35.

21. Demidenko G. V. and Uspenskii S. V. Partial Differential Equations and Systems not Solvable with Respect to the Highest-Order Derivative. New York, Basel: Marcel Dekker, 2003.

22. Kazakov A. L. and Spevak L. F. Numerical and analytical studies of a nonlinear parabolic equation with boundary conditions of a special form // Appl. Math. Modelling. 2013. V.37, N 10-11. P. 6918-6928.

23. Kazakov A. L. and Spevak L. F. An analytical and numerical study of a nonlinear parabolic equation with degeneration for the cases of circular and spherical symmetry // Appl. Math. Modelling. 2016. V.40, N 2. P.13331343.

24. Olver P. J. Direct reduction and differential constraints // Proc. Roy. Soc. Lond. Ser. A. 1994. V. 444, N 1922. P. 509-523.

25. Vazquez J. L. The Porous Medium Equation: Mathematical Theory. Oxford: Clarendon Press, 2007.

Казаков Александр Леонидович

Институт динамики систем и теории управления им. В. М. Матросова СО РАН, ул. Лермонтова, 134, Иркутск, 664033 РОССИЯ. E-mail: kazakov@icc.ru.
Поступила в редакцию 21 ноября 2018 г.

Получена после доработки 26 января 2019 г.

Принята к публикации 27 февраля 2019 г. 